\title{
An Explicit Formula for Two-Dimensional Singly-Periodic Regularized Stokeslets Flow Bounded by a Plane Wall
}

\author{
Forest O. Mannan ${ }^{1, *}$ and Ricardo Cortez ${ }^{1}$ \\ ${ }^{1}$ Department of Mathematics, Tulane University, New Orleans, \\ Louisiana 70118-5665, USA.
}

Received 22 December 2016; Accepted (in revised version) 13 April 2017

\begin{abstract}
We derive a closed form expression for the regularized Stokeslet in two space dimensions with periodic boundary conditions in the $x$-direction and a solid plane wall at $y=0$. To accommodate the no-slip condition on the wall, a system of images for the regularized Stokeslets was used. The periodicity is enforced by writing all elements of the image system in terms of a Green's function whose periodic extension is known. Although the formulation is derived in the context of regularized Stokeslets, the expression for the traditional (singular) Stokeslet is easily found by taking the limit as the regularization parameter approaches zero. The new formulation is validated by comparing results of two test problems: the Taylor infinite waving sheet and the motion of a cylinder moving near a wall. As an example of an application, we use our formulation to compute the motion and flow generated by cilia using a model that does not prescribe the motion so that the beat period and synchronization of neighboring cilia are a result of the forces developed along the cilia.
\end{abstract}

AMS subject classifications: 76D07, 76Z05

Key words: Regularized Stokeslets, singly-periodic flow, method of images.

\section{Introduction}

Spatially periodic systems have been used for many years to study phenomena with large numbers of particles or with repeated structures immersed in a viscous fluid. Examples are the dynamics of sedimenting particles [14,36], sedimentation of fibers [33], instabilities associated with some flows with periodicity [12] and with active suspensions [34], flow past arrays of spheres [18,43], and flows generated by arrays of cilia [42].

${ }^{*}$ Corresponding author. Email addresses: fmannan@tulane.edu (F. O. Mannan), rcortez@tulane.edu (R. Cortez) 
In cases of large numbers of particles in space, it becomes computationally prohibitive to account for each particle, so models with a smaller number of the particles in a periodic domain are used to capture the behavior of those systems. In most of these cases, the models begin with an infinite sum representing the contributions of the periodic replication of forces and proceed to truncate the sums based on rapidly converging formulations $[4,9,18,24,25,31,40]$. Since many of these flows can occur near a wall, as is the case of cilia attached to a surface, the swimming of sperm, the sedimentation of particles, and flow past obstacles $[16,22,35]$, there has been a need to develop methods to satisfy a no-flow condition at an infinite plane $[1,3]$ in otherwise unbounded domains.

There is continued interest in modeling two-dimensional Stokes flow in unbounded domains $[11,17]$ or with periodic boundary conditions either in both directions or only in one direction (see e.g. [6,27,31,34]). Marple et al. [27] recently considered the flow of vesicles in a channel by computing two dimensional Stokes flow with periodic boundary conditions at the channel outlet. Their approach is to use direct free-space summation that includes the two nearest periodic images and approximate the contributions from the more distant images using proxy sources on the boundary of an auxiliary domain. Bryngelson and Freund [6] used boundary integral methods to simulate two-dimensional channel flow with a dense suspension of deforming fluid-filled capsules. Crowdy and Or [11] developed a two-dimensional model to compute the motion of a swimmer near a wall using complex analysis. Importantly, their results show sufficient agreement with experiments and three-dimensional models to conclude that 2D models can provide insight into the dynamics of low-Reynolds number swimmers.

Here we describe an efficient method for two-dimensional flows generated by cilia or particle suspensions near a plane wall at $y=$ constant and with periodic boundary conditions in the $x$-direction (see Fig. 1). Our motivation comes from the motion of cilia, which are attached to a surface and beat to generate coordinated flows. The effect of multiple cilia beating next to each other can be modeled by imposing periodicity while the surface lined by the cilia is modeled as an infinite wall where no-flow boundary conditions are satisfied. We proceed by first adapting the method of regularized Stokeslets [8] for the case of periodic boundary conditions in the $x$-direction through the use of a singlyperiodic Green's function. We then derive the system of images $[1,10]$ that analytically cancels the flow at the wall. The result is an explicit formula for the flow due to regularized singly-periodic Stokeslets near a plane wall in two dimensions, which provides a straight forward way to investigate the flows of interest.

There have been many approaches to simulating cilia but they generally fall into two categories. Either the shape of the cilium beat is assumed to be known based on observed biological data $[20,23]$ or the motion of the cilium emerges from the model of an internal force generating mechanism $[7,15,16,28]$. While modeling the internal mechanisms is more challenging, it has the feature that the motion of the cilium itself is coupled to the fluid and is a priori unknown. With this approach, properties that depend on hydrodynamic coupling can be investigated, including how the beat frequency depends on the spacing between cilia and the synchronization of cilia beat patterns. In the present appli- 


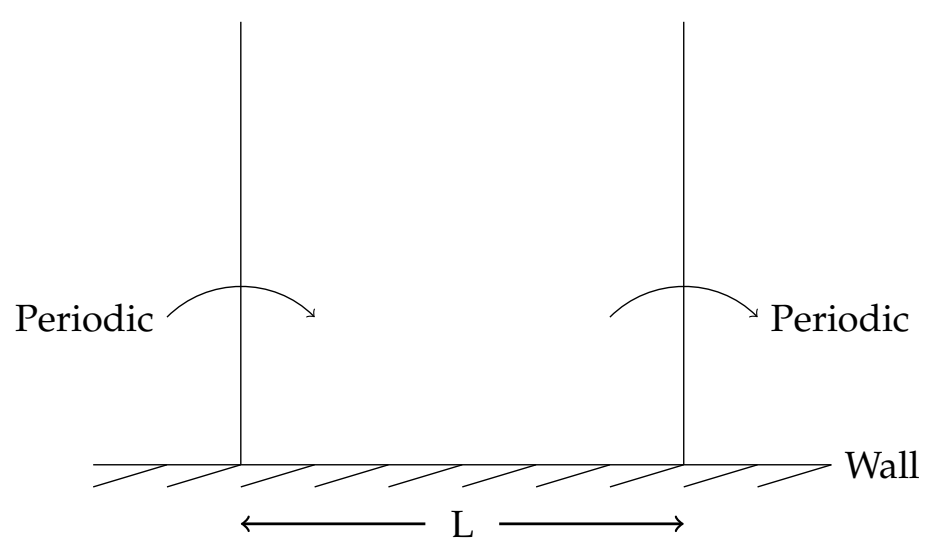

Figure 1: Schematic of the domain $[0, L) \times[w, \infty)$ where the Stokes equations are solved with periodic boundary conditions at $x=0$ and $x=L$, and zero flow conditions at $y=w$.

cation the internal mechanisms are modeled based on the work by Dillon and Fauci [13] in order to examine such properties. This is described in Section 3.3.

\section{Stokeslets in two dimensions}

\subsection{Free space Stokeslet}

The incompressible Stokes equations are

$$
0=-\nabla p+\mu \Delta \mathbf{u}+\mathbf{g} \delta\left(\mathbf{x}-\mathbf{x}_{0}\right), \quad 0=\nabla \cdot \mathbf{u},
$$

where $\mathbf{u}$ is the fluid velocity, $p$ is the fluid pressure, $\mu$ is the viscosity, $\mathbf{g}$ is an external constant force and $\delta\left(\mathbf{x}-\mathbf{x}_{0}\right)$ is the Dirac delta distribution centered at $\mathbf{x}_{0}$. The fundamental solution of the Stokes equations in $\mathbb{R}^{2}$ is called a Stokeslet and is given by (summation notation assumed)

$$
\begin{aligned}
& 4 \pi p=4 \pi g_{n} \frac{\partial}{\partial x_{n}} G_{0}(|\hat{\mathbf{x}}|), \\
& 4 \pi \mu u_{j}=4 \pi g_{n}\left[\frac{\partial^{2}}{\partial x_{j} \partial x_{n}}-\delta_{j n} \Delta\right] B_{0}(|\hat{\mathbf{x}}|),
\end{aligned}
$$

where the functions $B_{0}$ and $G_{0}$ are

$$
B_{0}(r)=\frac{r^{2}}{16 \pi}\left[\log \left(r^{2}\right)-3\right], \quad G_{0}(r)=\Delta B_{0}(r)=\frac{1}{4 \pi}\left[\log \left(r^{2}\right)-1\right]
$$


Specifically, the Stokeslet becomes

$$
\begin{aligned}
& 4 \pi p=2 g_{n} \frac{\hat{x}_{n}}{r^{2}}, \\
& 4 \pi \mu u_{j}(\mathbf{x})=g_{n}\left[\frac{\hat{x}_{j} \hat{x}_{n}}{r^{2}}-\delta_{j n} \log (r)\right],
\end{aligned}
$$

with $r=|\hat{\mathbf{x}}|$ and $\hat{\mathbf{x}}=\mathbf{x}-\mathbf{x}_{0}$ with components $\left(\hat{x}_{1}, \hat{x}_{2}\right)$. Note the singularity when $r=0$.

\subsection{Regularization of the Stokeslet}

One way to regularize the Stokeslet [8] is to modify the function $B(r)$ and apply the operator in Eq. (2.2) to define the incompressible velocity. For example, letting $0<\delta \ll 1$ and setting

$$
B_{\delta}(r)=\frac{\left(r^{2}+\delta^{2}\right)}{16 \pi}\left[\log \left(r^{2}+\delta^{2}\right)-3\right]
$$

we can compute

$$
G_{\delta}(r)=\Delta B_{\delta}(r)=\frac{1}{4 \pi}\left[\log \left(r^{2}+\delta^{2}\right)-1-\frac{\delta^{2}}{r^{2}+\delta^{2}}\right]
$$

so that using these functions in Eqs. (2.1)-(2.2) leads to the divergence-free regularized Stokeslet

$$
\begin{aligned}
& 2 \pi p=g_{n} \hat{x}_{n}\left(\frac{1}{r^{2}+\delta^{2}}+\frac{\delta^{2}}{\left(r^{2}+\delta^{2}\right)^{2}}\right), \\
& 4 \pi \mu u_{j}(\mathbf{x})=g_{n}\left[\frac{\hat{x}_{j} \hat{x}_{n}}{r^{2}+\delta^{2}}-\delta_{j n}\left(\frac{1}{2} \log \left(r^{2}+\delta^{2}\right)-\frac{\delta^{2}}{r^{2}+\delta^{2}}\right)\right] .
\end{aligned}
$$

\subsection{Flow bounded by a wall}

We are interested in developing an expression for the flow generated by a Stokeslet of strength $\mathbf{g}$ at $\mathbf{x}_{0}$ so that the fluid velocity vanishes at all points on the line $y=w$. The strategy is to place a Stokeslet at the image point $\mathbf{x}_{I}$ (see Fig. 2) plus additional elements that exactly cancel the flow at the wall. The image system is known for the singular Stokeslet [30] and for regularized Stokeslets in three dimensions [10]. The twodimensional case is similar so we provide only the main ideas here. The elements needed are a Stokes doublet, a potential dipole and rotlets.

\subsubsection{The Stokeslet velocity}

Based on Eq. (2.2), the velocity generated by a general regularized Stokeslet is

$$
4 \pi \mu u_{j}=4 \pi g_{n}\left[\frac{\partial^{2}}{\partial x_{j} \partial x_{n}}-\delta_{j n} \Delta\right] B_{\delta}(|\hat{\mathbf{x}}|),
$$




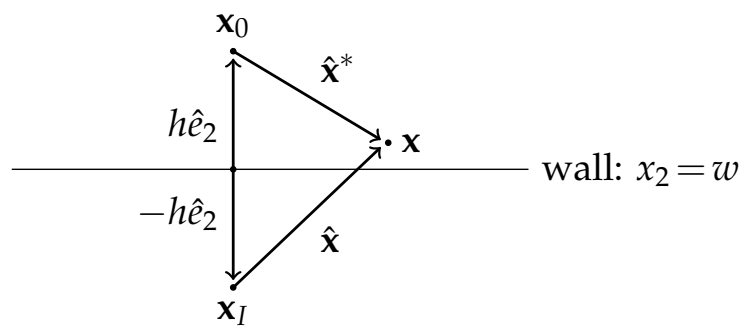

Figure 2: An illustration of the geometry and notation of the image system of a Stokeslet located at $\mathbf{x}_{0}$ which is a distance $h$ above a solid wall.

and it satisfies $\nabla \cdot \mathbf{u}=0$ analytically for any $B_{\delta}$. Taking advantage of the radial symmetry of $B_{\delta}$, the regularized Stokeslet in two dimensions can be written in vector form as

$$
S[\mathbf{g}](\mathbf{x})=\mathbf{g} H_{1}(r)+(\mathbf{g} \cdot \hat{\mathbf{x}}) \hat{\mathbf{x}} H_{2}(r),
$$

where $r=|\hat{\mathbf{x}}|, \hat{\mathbf{x}}=\mathbf{x}-\mathbf{x}_{0}$ and

$$
H_{1}(r)=-B_{\delta}^{\prime \prime}, \quad H_{2}(r)=\frac{r B_{\delta}^{\prime \prime}-B_{\delta}{ }^{\prime}}{r^{3}} .
$$

\subsubsection{The Stokes doublet velocity}

The Stokes doublet is obtained from taking the directional derivative of the Stokeslet in an arbitrary direction $\mathbf{b}$. Applying $(\mathbf{b} \cdot \nabla)$ to $S[\mathbf{g}]$ yields the following velocity

$$
S D[\mathbf{g}, \mathbf{b}]=(\mathbf{g} \cdot \mathbf{b}) \hat{\mathbf{x}} H_{2}(r)+(\mathbf{b} \cdot \hat{\mathbf{x}}) \mathbf{g} \frac{H_{1}^{\prime}(r)}{r}+(\mathbf{g} \cdot \hat{\mathbf{x}}) \mathbf{b} H_{2}(r)+(\mathbf{g} \cdot \hat{\mathbf{x}})(\mathbf{b} \cdot \hat{\mathbf{x}}) \hat{\mathbf{x}} \frac{H_{2}^{\prime}(r)}{r} .
$$

\subsubsection{The potential dipole velocity}

The potential dipole is obtained by taking the negative Laplacian of the Stokeslet. Doing so on a Stokeslet of strength $\mathbf{q}$ yields the velocity field

$$
P D[\mathbf{q}]=\mathbf{q} D_{1}(r)+(\mathbf{q} \cdot \hat{\mathbf{x}}) \hat{\mathbf{x}} D_{2}(r),
$$

where $G_{\delta}=\Delta B_{\delta}$ and

$$
D_{1}(r)=G_{\delta}^{\prime \prime}, \quad D_{2}(r)=-\frac{r G_{\delta}^{\prime \prime}-G_{\delta}^{\prime}}{r^{3}} .
$$

\subsubsection{The rotlet velocity}

A rotlet is the antisymmetric velocity field derived from a Stokes doublet, that is

$$
\frac{1}{2}(S D[\mathbf{g}, \mathbf{b}]-S D[\mathbf{b}, \mathbf{g}])=\frac{1}{2}\left(g_{2} b_{1}-g_{1} b_{2}\right)\left(\begin{array}{c}
-\hat{x}_{2} \\
\hat{x}_{1}
\end{array}\right)\left(H_{2}-\frac{H_{1}^{\prime}}{r}\right) .
$$

By defining $L=\frac{1}{2}\left(g_{2} b_{1}-g_{1} b_{2}\right)$ and simplifying the last factor, we arrive at the rotlet velocity

$$
R[L]=L \frac{G_{\delta}^{\prime}}{r}\left(\begin{array}{c}
-\hat{x}_{2} \\
\hat{x}_{1}
\end{array}\right) \text {. }
$$




\subsection{The image system for a regularized Stokeslet}

Assume a Stokeslet of strength $\mathbf{g}$ at an arbitrary location $\mathbf{x}_{0}$ above a horizontal wall located at $x_{2}=w$. The system of images will be obtained by adding various elements at the image point $\mathbf{x}_{I}=\mathbf{x}_{0}-2 h \mathbf{e}_{2}$ where $h$ is the height the Stokeslet is above the wall. In what follows $\hat{\mathbf{x}}^{*}$ refers to the vector from the location of the Stokeslet to an arbitrary point $\mathbf{x}$, and $\hat{\mathbf{x}}$ refers to the vector from the image point $\mathbf{x}_{I}$ to $\mathbf{x}$ (see Fig. 2). Additionally, $S^{*}[\cdot]$ represents the Stokeslet velocity at $\mathbf{x}_{0}$.

For an arbitrary point on the wall, $\mathbf{x}=(x, w)$ and we have that $\hat{\mathbf{x}}=\left(x-x_{0}, h\right)$ and $|\hat{\mathbf{x}}|=\left|\hat{\mathbf{x}}^{*}\right|$. Consider the velocity at $(x, w)$ due to the original Stokeslet of strength $\mathbf{g}$ at $\mathbf{x}_{0}$ and a second Stokeslet at $\mathbf{x}_{I}$ of strength $-\mathbf{g}$ :

$$
\begin{aligned}
S^{*}[\mathbf{g}]+S[-\mathbf{g}] & =\left[\left(\mathbf{g} \cdot \hat{\mathbf{x}}^{*}\right) \hat{\mathbf{x}}^{*}-(\mathbf{g} \cdot \hat{\mathbf{x}}) \hat{\mathbf{x}}\right] H_{2}(r) \\
& =\left[\left(\mathbf{g} \cdot\left(\hat{\mathbf{x}}-2 h \mathbf{e}_{2}\right)\right)\left(\hat{\mathbf{x}}-2 h \mathbf{e}_{2}\right)-(\mathbf{g} \cdot \hat{\mathbf{x}}) \hat{\mathbf{x}}\right] H_{2}(r) \\
& =\left[-2 h g_{2} \hat{\mathbf{x}}-2 h(\mathbf{g} \cdot \hat{\mathbf{x}}) \mathbf{e}_{2}+4 h^{2} g_{2} \mathbf{e}_{2}\right] H_{2}(r) \\
& =-2 h \hat{x}_{1} H_{2}(r)\left(\begin{array}{c}
g_{2} \\
g_{1}
\end{array}\right) .
\end{aligned}
$$

To cancel the remaining flow at the wall, consider the velocity at $\mathbf{x}=(x, w)$ due to the linear combination of a Stokes doublet and a potential dipole as given below:

$$
\begin{aligned}
S D\left[\mathbf{e}_{2}, \mathbf{b}\right]+P D\left[\frac{1}{2} h \mathbf{b}\right]= & h \mathbf{b}\left[H_{2}(r)+\frac{D_{1}(r)}{2}\right]+h(\mathbf{b} \cdot \hat{\mathbf{x}}) \hat{\mathbf{x}}\left[\frac{H_{2}^{\prime}(r)}{r}+\frac{D_{2}(r)}{2}\right] \\
& +b_{2} \hat{\mathbf{x}} H_{2}(r)+(\mathbf{b} \cdot \hat{\mathbf{x}}) \mathbf{e}_{2} \frac{H_{1}^{\prime}(r)}{r} .
\end{aligned}
$$

In the singular case, it is known that Eq. (2.11) and Eq. (2.12) cancel each other for a specific choice of $\mathbf{b}$. Cancellation in the regularized case can be achieved if the Potential Dipole is formed from a slightly different regularization than the one used in the Stokeslets. The algorithm is:

1. Functions for the Stokeslets and Stokes doublet

(a) Choose $B_{\delta}$ and set $G_{\delta}=\Delta B_{\delta}$

(b) Compute $H_{1}, H_{2}$ from Eq. (2.7)

2. Functions for the dipole

(a) Define $\tilde{G}_{\delta}=2 \frac{B_{\delta}{ }^{\prime}}{r}$

(b) Compute $D_{1}, D_{2}$ from Eq. (2.9) using $\tilde{G}_{\delta}$ 
With these choices the following identities can be established

$$
\begin{aligned}
& \frac{H_{1}^{\prime}(r)}{r}=H_{2}(r)-\frac{G_{\delta}{ }^{\prime}}{r}, \quad H_{2}(r)=\frac{\tilde{G}_{\delta}{ }^{\prime}}{2 r}, \\
& H_{2}(r)+\frac{1}{2} D_{1}(r)=\frac{G_{\delta}{ }^{\prime}}{r}-\frac{\tilde{G}_{\delta}{ }^{\prime}}{r}, \quad \frac{H_{2}^{\prime}(r)}{r}+\frac{D_{2}(r)}{2}=0 .
\end{aligned}
$$

Applying these identities to the sum of Eq. (2.11) and Eq. (2.12) gives

$$
\begin{aligned}
& S^{*}[\mathbf{g}]+S[-\mathbf{g}]+S D\left[\mathbf{e}_{2}, \mathbf{b}\right]+P D\left[\frac{1}{2} h \mathbf{b}\right] \\
= & \frac{G_{\delta}{ }^{\prime}}{r}\left(\begin{array}{c}
h b_{1} \\
-b_{1} \hat{x}_{1}
\end{array}\right)+\frac{\tilde{G}_{\delta}{ }^{\prime}}{r}\left(\begin{array}{c}
\frac{1}{2} b_{2} \hat{x}_{1}-h b_{1}-h \hat{x}_{1} g_{2} \\
-\frac{1}{2} h b_{2}+\frac{1}{2}\left(b_{1} \hat{x}_{1}+h b_{2}\right)-h \hat{x}_{1} g_{1}
\end{array}\right) .
\end{aligned}
$$

Defining $\mathbf{b}=2 h\left(-g_{1}, g_{2}\right)$, reduces it to

$$
S^{*}[\mathbf{g}]+S[-\mathbf{g}]+S D\left[\mathbf{e}_{2}, \mathbf{b}\right]+P D\left[\frac{1}{2} h \mathbf{b}\right]=2 h g_{1}\left[\frac{G_{\delta}{ }^{\prime}}{r}-\frac{\tilde{G}_{\delta}{ }^{\prime}}{r}\right]\left(\begin{array}{c}
-h \\
\hat{x}_{1}
\end{array}\right) .
$$

Recalling that $\hat{x}_{2}=h$ for an point at the image location, this is exactly the difference of two rotlets of strength $L=2 h g_{1}$ where one is formed using $G_{\delta}$ and one is formed using $\tilde{G}_{\delta}$. Thus, zero velocity at the wall will be achieved by adding $\tilde{R}\left[2 h g_{1}\right]-R\left[2 h g_{1}\right]$ so that the complete regularized image system for the Stokeslet is given by

$$
S^{*}[\mathbf{g}]+S[-\mathbf{g}]+S D\left[\mathbf{e}_{2}, \mathbf{b}\right]+P D\left[\frac{1}{2} h \mathbf{b}\right]+\tilde{R}\left[2 h g_{1}\right]-R\left[2 h g_{1}\right],
$$

where $\mathbf{b}=2 h\left(-g_{1}, g_{2}\right)$.

\subsection{The image system for a specific regularization}

\begin{tabular}{|c|c|}
\hline The functions for the Stokeslets and Stokes Doublet & The functions for the dipole \\
\hline$B_{\delta}=\frac{1}{16 \pi}\left(r^{2}+\delta^{2}\right)\left(\log \left(r^{2}+\delta^{2}\right)-3\right)$ & $\tilde{G_{\delta}}=\frac{1}{4 \pi} \log \left(r^{2}+\delta^{2}\right)-\frac{1}{2 \pi}$ \\
$G_{\delta}=\frac{1}{4 \pi} \log \left(r^{2}+\delta^{2}\right)-\frac{\delta^{2}}{4 \pi\left(r^{2}+\delta^{2}\right)}-\frac{1}{4 \pi}$ & \\
\hline
\end{tabular}

These lead to

$$
\begin{aligned}
& H_{1}=\frac{\delta^{2}}{4 \pi\left(r^{2}+\delta^{2}\right)}-\frac{1}{8 \pi} \log \left(r^{2}+\delta^{2}\right), \\
& H_{2}=\frac{1}{4 \pi\left(r^{2}+\delta^{2}\right)}
\end{aligned}
$$




$$
\begin{aligned}
& D_{1}=\frac{\delta^{2}}{\pi\left(r^{2}+\delta^{2}\right)^{2}}-\frac{1}{2 \pi\left(r^{2}+\delta^{2}\right)}, \\
& D_{2}=\frac{1}{\pi\left(r^{2}+\delta^{2}\right)^{2}}, \\
& \frac{G_{\delta}^{\prime}}{r}-\frac{\tilde{G}_{\delta}^{\prime}}{r}=\frac{\delta^{2}}{2 \pi\left(r^{2}+\delta^{2}\right)^{2}} .
\end{aligned}
$$

\subsection{The periodic extension of the regularized Stokeslet}

Following [30, p.94] we begin with Eq. (2.6) and use $\hat{x}_{1}^{2}=r^{2}-\hat{x}_{2}^{2}$, to write the regularized Stokeslet velocity as

$$
\begin{aligned}
& 4 \pi \mu u_{1}(\mathbf{x})=g_{1}+\frac{\hat{x}_{2}\left(g_{2} \hat{x}_{1}-g_{1} \hat{x}_{2}\right)}{r^{2}+\delta^{2}}-\frac{1}{2} g_{1} \log \left(r^{2}+\delta^{2}\right), \\
& 4 \pi \mu u_{2}(\mathbf{x})=\frac{\hat{x}_{2}\left(g_{1} \hat{x}_{1}+g_{2} \hat{x}_{2}\right)}{r^{2}+\delta^{2}}-g_{2}\left(\frac{1}{2} \log \left(r^{2}+\delta^{2}\right)-\frac{\delta^{2}}{r^{2}+\delta^{2}}\right),
\end{aligned}
$$

which is equal to

$$
\begin{aligned}
& 4 \pi \mu u_{1}=(1-\mathcal{G}) g_{1}+\hat{x}_{2}\left(-g_{1} \frac{\partial \mathcal{G}}{\partial \hat{x}_{2}}+g_{2} \frac{\partial \mathcal{G}}{\partial \hat{x}_{1}}\right), \\
& 4 \pi \mu u_{2}=\left(\delta \frac{\partial \mathcal{G}}{\partial \delta}-\mathcal{G}\right) g_{2}+\hat{x}_{2}\left(g_{1} \frac{\partial \mathcal{G}}{\partial \hat{x}_{1}}+g_{2} \frac{\partial \mathcal{G}}{\partial \hat{x}_{2}}\right)
\end{aligned}
$$

when written in terms of the Green's function

$$
\mathcal{G}\left(\hat{x}_{1}, \hat{x}_{2}, \delta\right)=\frac{1}{2} \log \left(\hat{x}_{1}^{2}+\hat{x}_{2}^{2}+\delta^{2}\right) .
$$

This formulation is useful because there is a straight-forward way to extend it to the case of periodic boundary conditions in the $x_{1}$-direction. We are interested in replacing $\mathcal{G}$ with the periodic Green's function

$$
\mathcal{G}^{P}\left(\hat{x}_{1}, \hat{x}_{2}, \delta\right)=\sum_{j=-\infty}^{\infty} \frac{1}{2} \log \left[\left(\hat{x}_{1}+L j\right)^{2}+\hat{x}_{2}^{2}+\delta^{2}\right] .
$$

Fortunately, this is computable from the known result (see $[2,32])$

$$
\sum_{j=-\infty}^{\infty} \log \left((x+2 \pi j)^{2}+\xi^{2}\right)=\log (\cosh (\xi)-\cos (x))
$$

so that

$$
\mathcal{G}^{P}\left(\hat{x}_{1}, \hat{x}_{2}, \delta\right)=\frac{1}{2} \log \left(\cosh \left(\frac{2 \pi}{L} \sqrt{\hat{x}_{2}^{2}+\delta^{2}}\right)-\cos \left(2 \pi \hat{x}_{1} / L\right)\right)+\frac{1}{2} \log \left(L^{2} / 2 \pi^{2}\right) .
$$


where the last term on the right is a constant that makes $\mathcal{G}\left(\hat{x}_{1}, \hat{x}_{2}, \delta\right)$ and $\mathcal{G}^{P}\left(\hat{x}_{1}, \hat{x}_{2}, \delta\right)$ match asymptotically near $\left(\hat{x}_{1}, \hat{x}_{2}\right)=(0,0)$ for any $\delta$. Therefore, the Stokeslet velocity with periodicity in the $x_{1}$-direction is given by Eqs. (2.18)-(2.19) using the periodic Green's function:

$$
\begin{aligned}
4 \pi \mu u_{1} & =\left(1-\mathcal{G}^{P}\right) g_{1}+\hat{x}_{2}\left(-g_{1} \frac{\partial \mathcal{G}^{P}}{\partial \hat{x}_{2}}+g_{2} \frac{\partial \mathcal{G}^{P}}{\partial \hat{x}_{1}}\right), \\
4 \pi \mu u_{2} & =\left(\delta \frac{\partial \mathcal{G}^{P}}{\partial \delta}-\mathcal{G}^{P}\right) g_{2}+\hat{x}_{2}\left(g_{1} \frac{\partial \mathcal{G}^{P}}{\partial \hat{x}_{1}}+g_{2} \frac{\partial \mathcal{G}^{P}}{\partial \hat{x}_{2}}\right) .
\end{aligned}
$$

Next we describe how to satisfy the zero-flow condition at a plane wall using the method of images and how to write the image system in terms of $\mathcal{G}^{P}$ to introduce periodicity.

\subsection{The periodic extension of the image system}

Now we would like to write the image system in terms of the function $\mathcal{G}=\frac{1}{2} \log \left(r^{2}+\delta^{2}\right)$ so that the periodic version can easily be obtained by replacing $\mathcal{G}(r, \delta)$ with its periodic version $\mathcal{G}^{P}\left(\hat{x}_{1}, \hat{x}_{2}, \delta\right)$ in Eq. (2.20). The expression for the Stokeslet has already been stated in Eqs. (2.18)-(2.19).

The Stokes doublet. Note that $S D\left[\mathbf{e}_{2}, \mathbf{b}\right]=(\mathbf{b} \cdot \nabla) S\left[\mathbf{e}_{2}\right]$, so the relevant Stokes doublet is

$$
\begin{aligned}
& 4 \pi \mu u_{1}=(\mathbf{b} \cdot \nabla)\left(\hat{x}_{2} \frac{\partial \mathcal{G}}{\partial \hat{x}_{1}}\right), \\
& 4 \pi \mu u_{2}=(\mathbf{b} \cdot \nabla)\left(\delta \frac{\partial \mathcal{G}}{\partial \delta}-\mathcal{G}\right)+(\mathbf{b} \cdot \nabla)\left(\hat{x}_{2} \frac{\partial \mathcal{G}}{\partial \hat{x}_{2}}\right),
\end{aligned}
$$

or

$$
\begin{aligned}
& 4 \pi \mu u_{1}=b_{1} \hat{x}_{2} \frac{\partial^{2} \mathcal{G}}{\partial \hat{x}_{1}^{2}}+b_{2}\left(\frac{\partial \mathcal{G}}{\partial \hat{x}_{1}}+\hat{x}_{2} \frac{\partial^{2} \mathcal{G}}{\partial \hat{x}_{1} \partial \hat{x}_{2}}\right), \\
& 4 \pi \mu u_{2}=b_{1}\left(\delta \frac{\partial^{2} \mathcal{G}}{\partial \delta \partial \hat{x}_{1}}+\hat{x}_{2} \frac{\partial^{2} \mathcal{G}}{\partial \hat{x}_{1} \partial \hat{x}_{2}}-\frac{\partial \mathcal{G}}{\partial \hat{x}_{1}}\right)-b_{2} \hat{x}_{2} \frac{\partial^{2} \mathcal{G}}{\partial \hat{x}_{1}^{2}} .
\end{aligned}
$$

The dipole. For the dipole we use $\hat{x}_{1}^{2}=r^{2}-\hat{x}_{2}^{2}$ to write

$$
\begin{aligned}
& \mu u_{1}=D_{1} q_{1}+\hat{x}_{1}^{2} q_{1} D_{2}+\hat{x}_{1} \hat{x}_{2} q_{2} D_{2}=\left(D_{1}+r^{2} D_{2}\right) q_{1}+\hat{x}_{2}\left(-q_{1} \hat{x}_{2} D_{2}+q_{2} \hat{x}_{1} D_{2}\right), \\
& \mu u_{2}=D_{1} q_{2}+\hat{x}_{1} \hat{x}_{2} q_{1} D_{2}+\hat{x}_{2}^{2} q_{2} D_{2}=D_{1} q_{2}+\hat{x}_{2}\left(q_{1} \hat{x}_{1} D_{2}+q_{2} \hat{x}_{2} D_{2}\right),
\end{aligned}
$$

and use the identities

$$
D_{1}+r^{2} D_{2}=\frac{1}{4 \pi} \frac{2}{\delta} \frac{\partial \mathcal{G}}{\partial \delta}, \quad D_{1}=-\frac{2}{4 \pi} \frac{\partial^{2} \mathcal{G}}{\partial \delta^{2}}, \quad \hat{x}_{j} D_{2}=-\frac{1}{4 \pi} \frac{2}{\delta} \frac{\partial^{2} \mathcal{G}}{\partial \delta \partial \hat{x}_{j}}
$$


to get

$$
\begin{aligned}
& 4 \pi \mu u_{1}=\frac{2}{\delta} \frac{\partial \mathcal{G}}{\partial \delta} q_{1}+\frac{2 \hat{x}_{2}}{\delta}\left(q_{1} \frac{\partial^{2} \mathcal{G}}{\partial \delta \partial \hat{x}_{2}}-q_{2} \frac{\partial^{2} \mathcal{G}}{\partial \delta \partial \hat{x}_{1}}\right), \\
& 4 \pi \mu u_{2}=-2 \frac{\partial^{2} \mathcal{G}}{\partial \delta^{2}} q_{2}-\frac{2 \hat{x}_{2}}{\delta}\left(q_{1} \frac{\partial^{2} \mathcal{G}}{\partial \delta \partial \hat{x}_{1}}+q_{2} \frac{\partial^{2} \mathcal{G}}{\partial \delta \partial \hat{x}_{2}}\right) .
\end{aligned}
$$

Since $\mathcal{G}(r, \delta)$ is symmetric about the two arguments, its derivatives with respect to $\delta$ can be related to derivatives with respect to $\hat{x}_{1}$ and $\hat{x}_{2}$. Using the property

we get

$$
\frac{\hat{x}_{2}}{\delta} \frac{\partial \mathcal{G}}{\partial \delta}=\frac{\partial \mathcal{G}}{\partial \hat{x}_{2}}
$$

$$
\begin{aligned}
& 4 \pi \mu u_{1}=2 q_{1}\left(\frac{\partial^{2} \mathcal{G}}{\partial \hat{x}_{2}^{2}}\right)-2 q_{2}\left(\frac{\partial^{2} \mathcal{G}}{\partial \hat{x}_{1} \partial \hat{x}_{2}}\right), \\
& 4 \pi \mu u_{2}=2 q_{2}\left(\frac{\partial^{2} \mathcal{G}}{\partial \hat{x}_{1}^{2}}\right)-2 q_{1}\left(\frac{\partial^{2} \mathcal{G}}{\partial \hat{x}_{1} \partial \hat{x}_{2}}\right) .
\end{aligned}
$$

The rotlets. The velocity due to the difference of rotlets is

$$
\begin{aligned}
& \mu u_{1}=-L \hat{x}_{2}\left(\frac{G_{\delta}{ }^{\prime}}{r}-\frac{\tilde{G}_{\delta}{ }^{\prime}}{r}\right), \\
& \mu u_{2}=L \hat{x}_{1}\left(\frac{G_{\delta}{ }^{\prime}}{r}-\frac{\tilde{G}_{\delta}{ }^{\prime}}{r}\right) .
\end{aligned}
$$

Using the identity

$$
\hat{x}_{j}\left(\frac{G_{\delta}{ }^{\prime}}{r}-\frac{\tilde{G}_{\delta}{ }^{\prime}}{r}\right)=-\frac{1}{4 \pi} \delta \frac{\partial^{2} \mathcal{G}}{\partial \delta \partial \hat{x}_{j}},
$$

we can write

$$
\begin{aligned}
& 4 \pi \mu u_{1}=L \delta \frac{\partial^{2} \mathcal{G}}{\partial \delta \partial \hat{x}_{2}} \\
& 4 \pi \mu u_{2}=-L \delta \frac{\partial^{2} \mathcal{G}}{\partial \delta \partial \hat{x}_{1}} .
\end{aligned}
$$

\subsection{The full image system}

The full image system $S^{*}[\mathbf{g}]-S[\mathbf{g}]+S D\left[\mathbf{e}_{2}, \mathbf{b}\right]+P D\left[\frac{h}{2} \mathbf{b}\right]+\tilde{R}\left[2 h g_{1}\right]-R\left[2 h g_{1}\right]$ with $\mathbf{b}=$ $2 h\left(-g_{1}, g_{2}\right)$, after simplification, becomes

$$
\begin{aligned}
4 \pi \mu u_{1}= & -\mathcal{G}^{*} g_{1}+\hat{x}_{2}^{*}\left(-g_{1} \frac{\partial \mathcal{G}^{*}}{\partial \hat{x}_{2}}+g_{2} \frac{\partial \mathcal{G}^{*}}{\partial \hat{x}_{1}}\right) \\
& +\mathcal{G} g_{1}+\hat{x}_{2} g_{1} \frac{\partial \mathcal{G}}{\partial \hat{x}_{2}}+2 h g_{1}\left(\hat{x}_{2}-h\right) \frac{\partial^{2} \mathcal{G}}{\partial \hat{x}_{2}^{2}}+g_{2}\left(2 h-\hat{x}_{2}\right) \frac{\partial \mathcal{G}}{\partial \hat{x}_{1}}+2 h g_{2}\left(\hat{x}_{2}-h\right) \frac{\partial^{2} \mathcal{G}}{\partial \hat{x}_{1} \partial \hat{x}_{2}}
\end{aligned}
$$




$$
\begin{aligned}
4 \pi \mu u_{2}= & \left(\delta \frac{\partial \mathcal{G}^{*}}{\partial \delta}-\mathcal{G}^{*}\right) g_{2}+\hat{x}_{2}^{*}\left(g_{1} \frac{\partial \mathcal{G}^{*}}{\partial \hat{x}_{1}}+g_{2} \frac{\partial \mathcal{G}^{*}}{\partial \hat{x}_{2}}\right)+\left(\mathcal{G}-\delta \frac{\partial \mathcal{G}}{\partial \delta}-\hat{x}_{2} \frac{\partial \mathcal{G}}{\partial \hat{x}_{2}}\right) g_{2} \\
& -2 h g_{2}\left(\hat{x}_{2}-h\right) \frac{\partial^{2} \mathcal{G}}{\partial \hat{x}_{1}^{2}}+g_{1}\left(2 h-\hat{x}_{2}\right) \frac{\partial \mathcal{G}}{\partial \hat{x}_{1}}-2 h g_{1}\left(\hat{x}_{2}-h\right) \frac{\partial^{2} \mathcal{G}}{\partial \hat{x}_{1} \partial \hat{x}_{2}} .
\end{aligned}
$$

The periodicity in the $x$-direction is achieved by simply replacing $\mathcal{G}$ with the Green's function $\mathcal{G}^{P}\left(\hat{x}_{1}, \hat{x}_{2}, \delta\right)$ in Eq. (2.20).

\subsection{The far field velocity of the periodic image system}

The far field velocity due to a single force $\mathbf{g}=\left(g_{1}, g_{2}\right)$ can be calculated directly by taking the limit as $\hat{x}_{2} \rightarrow \infty$ of the periodic image system. This yields

$$
4 \pi \mu\left(u_{1}, u_{2}\right)=\left(g_{1} \frac{4 \pi h}{L}, 0\right)
$$

which shows that the flow far above the wall reaches a nonzero constant parallel to the wall.

\section{Numerical examples}

\subsection{Taylor's infinite waving sheet}

In Taylor's classic paper [38], the swimming velocity of an infinite sheet undergoing inextensible traveling waves of the parametric form $x(s)=s$ and $y(s)=b \sin (k s-\sigma t)$ is derived asymptotically. For small values of the wave amplitude $b$, this translational velocity in the $x$-direction is found to be

$$
\frac{\sigma}{2} b^{2} k\left(1-\frac{19}{16} b^{2} k^{2}+\cdots\right)
$$

As a way of validating the singly-periodic formulation of the regularized Stokeslets without a bounding plane (no images), we let the periodic box of length $L=2 \pi$ consist of a single period of the sheet so that the velocity at a point $\mathbf{X}\left(s_{0}\right)$ on the sheet can be expressed as

$$
\mathbf{u}\left(\mathbf{X}\left(s_{0}\right)\right)=\mathbf{U}+\int_{\Gamma} S^{P}[\mathbf{f}(s)]\left(\mathbf{X}\left(s_{0}\right)-\mathbf{X}(s)\right) d s,
$$

where $\mathbf{X}(s)$ is the arc length parameterization of the swimming sheet $\Gamma$ and $S^{P}[\mathbf{f}(s)](\cdot)$ is the periodic Stokeslet in Eqs. (2.21)-(2.22) and $\mathcal{G}^{\mathcal{P}}$ given in Eq. (2.20). Here, $\mathbf{u}\left(\mathbf{X}\left(s_{0}\right)\right)$ is the prescribed velocity of the traveling wave at $\mathbf{X}\left(s_{0}\right)$ and $\mathbf{U}$ is a constant translational velocity resulting from the motion. The integral is approximated with the composite 
midpoint rule. Under the constraint that the net force is zero, this yields the following linear system

$$
\left(\begin{array}{ccccc}
w_{1} \mathbf{S}^{P}\left(\mathbf{x}_{1}-\mathbf{x}_{1}\right) & w_{2} \mathbf{S}^{P}\left(\mathbf{x}_{1}-\mathbf{x}_{2}\right) & \cdots & w_{N} \mathbf{S}^{P}\left(\mathbf{x}_{1}-\mathbf{x}_{N}\right) & \mathbf{I} \\
w_{1} \mathbf{S}^{P}\left(\mathbf{x}_{2}-\mathbf{x}_{1}\right) & w_{2} \mathbf{S}^{P}\left(\mathbf{x}_{2}-\mathbf{x}_{2}\right) & \cdots & w_{N} \mathbf{S}^{P}\left(\mathbf{x}_{2}-\mathbf{x}_{N}\right) & \mathbf{I} \\
\vdots & & & & \\
w_{1} \mathbf{S}^{P}\left(\mathbf{x}_{N}-\mathbf{x}_{1}\right) & \cdots & & w_{N} \mathbf{S}^{P}\left(\mathbf{x}_{N}-\mathbf{x}_{N}\right) & \mathbf{I} \\
w_{1} \mathbf{I} & \cdots & & w_{N} \mathbf{I} & \mathbf{0}
\end{array}\right)\left(\begin{array}{c}
\mathbf{f}_{1} \\
\mathbf{f}_{2} \\
\vdots \\
\mathbf{f}_{N} \\
\mathbf{U}
\end{array}\right)=\left(\begin{array}{c}
\mathbf{u}_{1} \\
\mathbf{u}_{2} \\
\vdots \\
\mathbf{u}_{N} \\
\mathbf{0}
\end{array}\right)
$$

where the wave has been divided into $N$ equal subintervals and $w_{i}$ is the arc length of the $i$ th section. Each matrix element shown is a $2 \times 2$ block that provides the contribution of the force at one point to the velocity at another and $\mathbf{u}_{j}$ is the prescribed velocity at $\mathbf{x}_{j}$. This system can be solved for the unknown forces and the swimming velocity $\mathbf{U}$.

We set $\sigma=1, k=1$ and $\delta=0.7 \Delta x$ where $\Delta x=\frac{2 \pi}{k N}$ and compute the difference between the swimming velocity (in the $x$-direction) predicted by Taylor and the value found using periodic Stokeslets for several values of the amplitude $b$. This difference in swimming speed is not expected to vanish for finer discretizations since neither method gives the exact solution; however, we can write

$$
U_{\text {Taylor }}-U_{\text {Stokeslet }}=\left(U_{\text {Taylor }}-U_{\text {Exact }}\right)-\left(U_{\text {Stokeslet }}-U_{\text {Exact }}\right)=\epsilon(b)-\left(U_{\text {Stokeslet }}-U_{\text {Exact }}\right),
$$

where $\epsilon(b)$ is the asymptotic error in Taylor's swimming velocity, which depends on $b$. Thus if the periodic Stokeslets velocity converges to the exact velocity, the difference between the velocity predicted by Taylor and that obtained from periodic Stokeslets should approach the constant $\epsilon(b)$. Table 1 shows the results for several values of $b$ and different discretizations. In each column, the difference approaches a constant as the error in the Stokeslet computation becomes negligible compared to $\epsilon(b)$.

Table 1: The difference $U_{\text {Taylor }}-U_{\text {Stokeslets }}$ calculated with periodic Stokeslets for different wave amplitude heights $b$ and number of discretization points $N$. All data shown is with the parameters $\sigma=k=1, \delta=0.7 \Delta x$ and $\Delta x=\frac{2 \pi}{k N}$. As should be expected, the value that is approached is larger for larger $b$.

\begin{tabular}{||c|c|c|c|c|c||}
\hline$N$ & $b=0.05$ & $b=0.1$ & $b=0.2$ & $b=0.4$ & $b=0.8$ \\
\hline $2^{5}$ & 0.00015887 & 0.00062111 & 0.00223645 & 0.00395793 & -0.10447483 \\
$2^{6}$ & 0.00008407 & 0.00032506 & 0.00110050 & 0.00009622 & -0.11281151 \\
$2^{7}$ & 0.00004310 & 0.00016295 & 0.00047932 & -0.00201021 & -0.11743034 \\
$2^{8}$ & 0.00002161 & 0.00007794 & 0.00015390 & -0.00311139 & -0.11985732 \\
$2^{9}$ & 0.00001060 & 0.00003439 & -0.00001274 & -0.00367452 & -0.12110090 \\
$2^{10}$ & 0.00000503 & 0.00001234 & -0.00009707 & -0.00395930 & -0.12173032 \\
$2^{11}$ & 0.00000222 & 0.00000125 & -0.00013949 & -0.00410251 & -0.12204694 \\
$2^{12}$ & 0.00000082 & -0.00000432 & -0.00016077 & -0.00417431 & -0.12220573 \\
$2^{13}$ & 0.00000011 & -0.00000710 & -0.00017142 & -0.00421026 & -0.12228525 \\
\hline
\end{tabular}




\subsection{Periodic flow past obstacles near a wall}

Here we consider examples in the semi-infinite domain $(x, y) \in\left[-\frac{L}{2}, \frac{L}{2}\right] \times[0, \infty)$ with periodic boundary conditions in $x$ and zero flow at $y=0$. A case of a background flow over solid stationary obstacles is depicted in Fig. 3, where the parabolic flow $\mathbf{U}=y(2.5 \pi-y) \mathbf{e}_{1}$ is perturbed by the presence of fixed circular solid obstacles randomly placed. Forces on the surface of the circles are computed so that their induced velocity exactly counters the background flow. As in the previous example, a linear system is constructed for the forces except that in this case there is no need to enforce a zero net force constraint. Once the forces are found, they are used to compute the fluid velocity everywhere. Fig. 3 shows streamlines of the flow over two periods of the above domain solved with a discretization size of $\Delta s=\pi / 250$ (dimensionless units) on the circles and a regularization parameter of $\delta=\Delta s$.

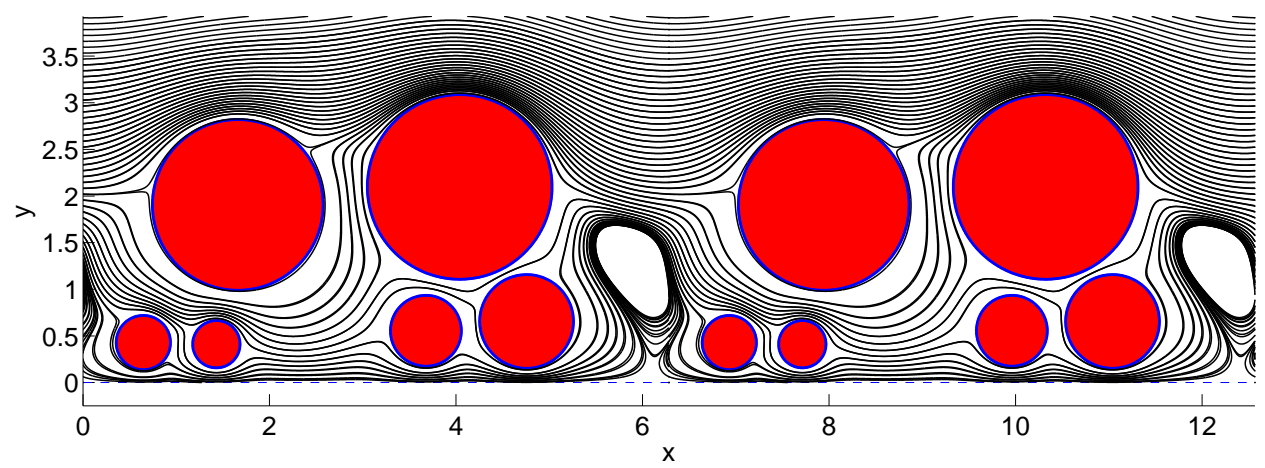

Figure 3: A visualization of the streamlines induced by a parabolic background flow in a periodic domain past fixed circular obstacles. Note that 2 periods are shown.

As a way of assessing our method, we turn to the problem of computing the force $\left(F_{x}, F_{y}\right)$ exerted by a single cylinder moving with speed $U$ parallel to the wall and $V$ perpendicular to it. In the nonperiodic case $(L \rightarrow \infty)$, Jeffrey and Onishi [21] provide the formulas

$$
\begin{aligned}
\frac{F_{x}}{\mu U} & =\frac{4 \pi}{\log \left(d+\sqrt{d^{2}-r^{2}}\right)-\log (r)}, \\
\frac{F_{y}}{\mu V} & =\frac{4 \pi}{\log \left(d+\sqrt{d^{2}-r^{2}}\right)-\log (r)-\sqrt{d^{2}-r^{2}} / d}
\end{aligned}
$$

for the corresponding forces by a cylinder of radius $r$ whose center is at a distance $d$ from the wall. Note that when the cylinder is very close to the wall, $F_{x} \sim(d / r-1)^{-1 / 2}$ and $F_{y} \sim(d / r-1)^{-3 / 2}$. Fig. 4 shows the force components for $r=0.25$, a discretization size $\Delta s=1 / 1600$ on the cylinder surface, a regularization parameter $\delta=\Delta s$ and several values of the periodic box length $L$. The figure shows that the effect of periodicity is to 

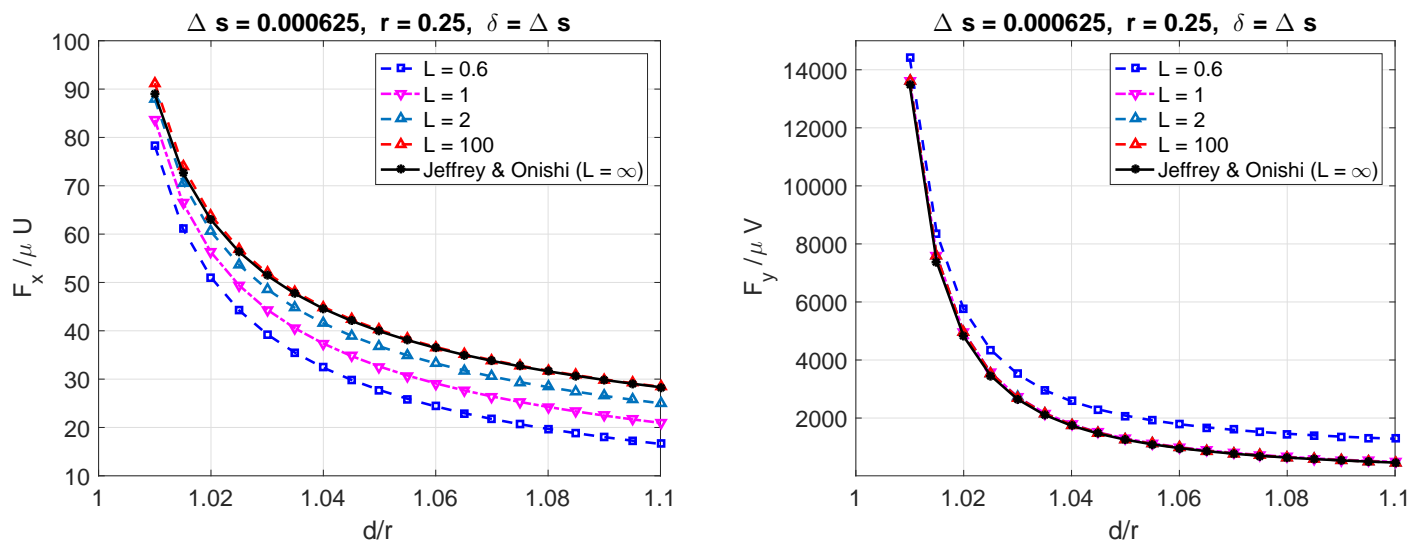

Figure 4: Forces on a cylinder translating parallel (left) and perpendicular (right) to the wall for various sizes of the periodic box $L$.

reduce the net tangential force $F_{x}$ on the cylinder and that when $L \geq 100$, periodicity has very little influence on the resulting force as the curves collapse onto the predicted values in [21]. In fact, the force $F_{y}$ perpendicular to the wall seems to be nearly independent of the periodic effects except when $L<1=4 r$ which requires larger forces.

The effect of the discretization parameter $\Delta s$ and the regularization parameter $\delta$ can be seen in Fig. 5, which shows the relative error in the force computation when $L=100$ compared to the values in [21]. The choice $\delta=\Delta s$ gave consistently better results than larger values of $\delta$ for a range of discretizations. The figure also shows that the errors in the force computations are larger when the gap between the cylinder and the wall is smaller, as one would expect due to the large velocity gradients that develop within the gap.

\subsection{Modeling cilia}

Cilia play a diverse and important role in biology, from facilitating the transport of the ovum through the Fallopian tubes to the movement of mucus and particles in the lungs and mechanotransduction $[19,37,39]$. Many of these motile cilia occur in groups extending from the surface of a cell. A natural idealization is to treat the cell surface as a wall and assume the cilia are periodic along the wall. Under these assumptions, the proposed numerical method is applicable. The biological structure of a cilium has been well documented (the "9+2" axoneme structure), but it is still unknown how the local mechanisms combine to form a cohesive and regular beat [26]. A model that imposes a prescribed beat form cannot be used to study switching mechanisms that signal the end of a power stroke and the beginning of the recovery stroke. For this, the approach adopted here is that of Dillon and Fauci $[13,42]$, where specific assumptions about how the internal structure operates are made and the beat pattern is allowed to emerge. 

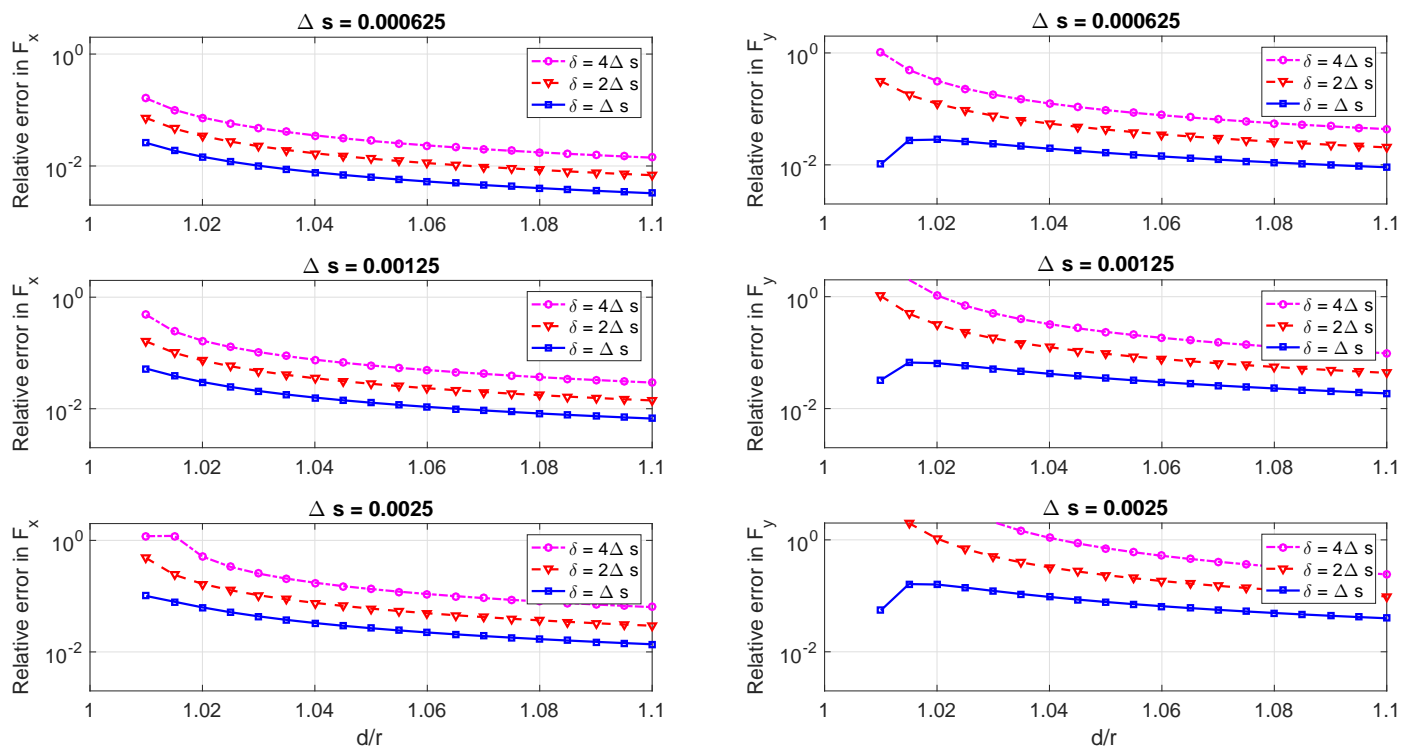

Figure 5: Relative errors in the net force needed to translate a cylinder parallel (left) and perpendicular (right) to a wall. The periodic box size was set to $L=100$ in order to compare with the values given by Jeffrey and Onishi.

\subsubsection{A model of the cilium structure}

Each cilium is assumed to be composed of two microtubule pairs. Each microtubule pair is represented by two filaments attached with diagonal cross links (see Fig. 6). The microtubule pairs are connected to each other by horizontal and diagonal links between the inner filaments representing nexins and dyneins, respectively. The diagonal dyneins are responsible for creating the bending of the cilium. Bending in both directions, and thus the whole ciliary beat, is achieved by assuming there are two sets of dyneins which are alternatingly activated. In the LR mode (short for "left-to-right"), a set of dyneins oriented diagonally downwards from left to right creates the power stroke. The contraction of the LR dyneins pulls the left microtubule down and the right microtubule up and effect an overall bending to the right since the base is anchored to the wall. Analogously, in the RL mode, a set of dyneins oriented diagonally upwards from left to right creates the recovery stroke. In both instances the other set of dyneins is deactivated. The horizontal nexins act to enforce an overall spacing between the microtubule pairs.

The entire structure and cross links are modeled as springs attached to different nodes along the microtubules. The horizontal nexin connections are permanently attached to nodes on the right inner filament and dynamically attach to the closest node on the left inner filament irrespective of mode. In the LR mode the dyneins are permanently attached to the left filament and in the RL mode they are permanently attached to the right filament. In either case the dynamic side attaches two nodes below the nearest node on 


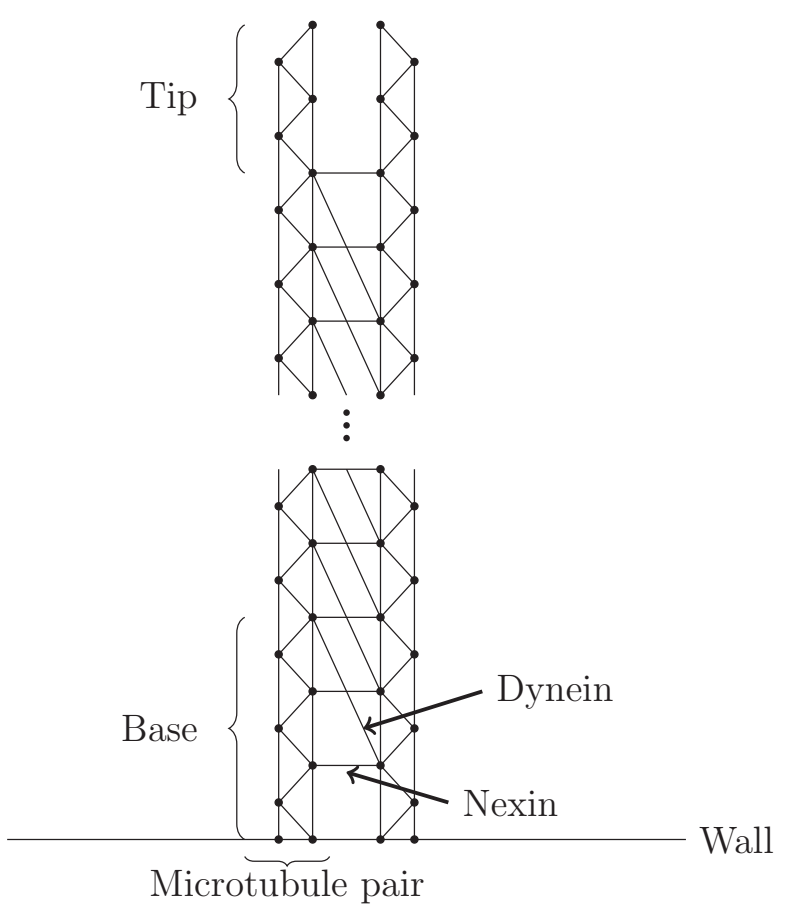

Figure 6: Cilium in LR mode: dyneins are permanently attached on the left inner filament and dynamically connect two nodes below the closest node on the right inner filament.

the opposite filament. In the first sections of the cilium, designated as the base, there are no dyneins. Additionally, in the last segments of the cilium, designated as the tip, there are no dyneins nor nexin connections between the microtubules. See [26] for a complete description.

In this application, the flow is driven by spring forces. Assuming springs connect node $\mathbf{x}_{j}$ to other nodes, the force per unit length generated at the node $\mathbf{x}_{j}$ is

$$
\mathbf{f}_{j}=-\sum_{i} S_{j i}\left(\left\|\mathbf{x}_{j}-\mathbf{x}_{i}\right\|-R_{j i}\right) \frac{\mathbf{x}_{j}-\mathbf{x}_{i}}{\left\|\mathbf{x}_{j}-\mathbf{x}_{i}\right\|}
$$

where $S_{j i}$ is the stiffness constant of the spring between $\mathbf{x}_{j}$ and $\mathbf{x}_{i}$, and $R_{j i}$ is the spring rest length. The Stokes equations are

$$
0=-\nabla p+\mu \Delta \mathbf{u}+\mathbf{F}, \quad \nabla \cdot \mathbf{u}=0,
$$

where $\mathbf{F}(\mathbf{x})=\sum_{j} \mathbf{f}_{j} \phi_{\delta}\left(\mathbf{x}-\mathbf{x}_{j}\right) \Delta s$ is a discretization of a line integral along a filament with nodes separated by a distance $\Delta s$.

For the simulations, we wrote the equations in dimensionless variables based on a representative length scale $\ell$ and velocity scale $U$. The force and pressure were scaled by $\mathcal{F}=\mu U / \ell^{2}$ and $\mathcal{P}=\ell \mathcal{F}$, respectively. This leads to the dimensionless model

$$
0=-\nabla p^{*}+\Delta \mathbf{u}^{*}+\mathbf{F}^{*}, \quad \nabla \cdot \mathbf{u}^{*}=0,
$$


where

$$
\mathbf{F}^{*}\left(x^{*}\right)=\sum_{j} \mathbf{f}_{j}^{*} \phi_{\delta^{*}}\left(\mathbf{x}^{*}-\mathbf{x}_{j}^{*}\right) \Delta s^{*}, \quad \mathbf{f}_{j}^{*}=-\sum_{i} S_{j i}^{*}\left(\left\|\mathbf{x}_{j}^{*}-\mathbf{x}_{i}^{*}\right\|-R_{j i}^{*}\right) \frac{\mathbf{x}_{j}^{*}-\mathbf{x}_{i}^{*}}{\left\|\mathbf{x}_{j}^{*}-\mathbf{x}_{i}^{*}\right\|}
$$

and

$$
S_{j i}^{*}=\ell^{2} S_{j i} / \mu U, \quad \mathbf{f}_{j}^{*}=\ell \mathbf{f}_{j} / \mu U .
$$

The representative scales used were $\ell=1 \mu \mathrm{m}$ and $U=1000 \mu \mathrm{m} / \mathrm{s}$ (based on a $14 \mu \mathrm{m}$ cilium beating at about $10 \mathrm{~Hz}$ ).

The dyneins and diagonal connections have variable spring constants. The diagonal springs are stronger at the base to prevent kinking of the cilium. Their spring constants decay linearly from $1.5 \times 10^{3}$ at the base to $10^{3}$ at $1 / 6$ of the length of each filament, and are $10^{3}$ thereafter. The dynein spring constant changes dynamically based on the displacement between the nodes as

$$
k \begin{cases}0, & \text { for } z \leq 0.7 d \\ (z-0.7 d) / 0.3 d, & \text { for } 0.7 d \leq z \leq d \\ 1, & \text { for } z>d\end{cases}
$$

where $z=\left\|\mathbf{x}_{j}-\mathbf{x}_{i}\right\|, d$ is the initial horizontal distance between the filaments in the microtubule pair, and $k$ is a constant to be given as explained below.

To create a net fluid movement in a Stokes flow, a nonsymmetric beat pattern is required. This is achieved by activating the recovery and power stroke dyneins differently. In this model the power dyneins are all given the same spring constant, $k=1.5$, but the recovery dyneins are given spring constants $k$ that decrease linearly from 3 at the base to 0 at the tip. In either stroke all of the power or recovery dyneins are respectively activated. Note that this is a modification of the model being replicated given in [13] where the recovery dyneins all have the same spring constant and are only activated up to a certain height controlled by a curvature based algorithm. Also, the model in [13] enforces zero flow at a given height above the cilium by virtue of imposing periodic boundary conditions in both coordinate directions and zero flow at the wall. This is not the case in the "open top" domain considered here where there is a constant net flow above the cilium given by Eq. (2.23). The present choice of stiffness constants was made because it led to more stable power and recovery stroke cycles.

The switching between power strokes and recovery strokes is done by keeping track of how far one microtubule end slides distally past the other. The RL mode is activated once the right microtubule extended a specified number of nodes beyond the tip of the left microtubule. Similarly, the LR mode is activated when the left microtubule extends a specified number of nodes beyond the tip of the right microtubule. The same switching distances were not used for all the simulations and the specific switching distances are stated as their results are discussed below.

The initial spacing, spring constants and height for all simulations are given in Table 2. With these parameters and a sliding distance of 9 nodes set to switch from LR to RL 
Table 2: Dimensionless parameters used in the $2 \mathrm{~d}$ cilium structure.

\begin{tabular}{||lc||}
\hline Power dynein stiffness & 1.5 \\
Max recovery dynein stiffness & 3 \\
Nexin stiffness & 110 \\
Vertical filament stiffness & $10^{4}$ \\
Diagonal stiffness & $10^{3}$ \\
Distance between microtubules & 0.35 \\
Distance between filaments & 0.117 \\
Vertical distance between nodes & 0.117 \\
Height of cilium & 14.04 \\
Number of nodes in filament & 121 \\
Regularization parameter, $\delta$ & 0.234 \\
\hline
\end{tabular}

modes and a sliding distance of 8 nodes set to switch from RL to LR modes an example of the resulting beat pattern is given Fig. 7(a).

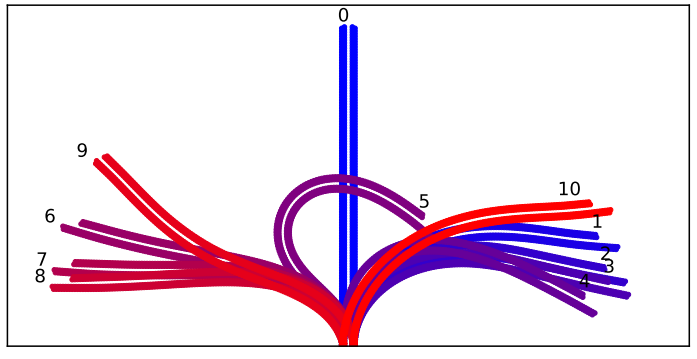

(a)

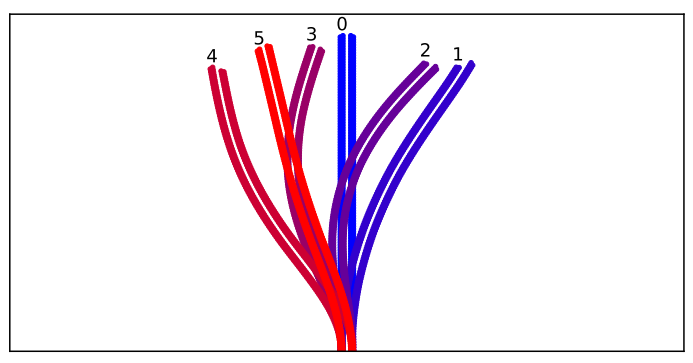

(b)

Figure 7: (a) Snapshots from the beat cycle of a cilium with periodic spacing of 20 and a sliding distance of 9 nodes and 8 nodes to switch from LR and RL modes respectively. (b) Snapshots from the beat cycle of a cilium with a sliding distance of 5 and 3 nodes respectively. In both figures the numbers notate the order of snapshots and the colors vary from blue to red and the opacity decreases as time progresses.

\subsubsection{Results}

Our integrative model of ciliary beating that is coupled with the surrounding fluid lends itself to investigating how the beat frequency, fluid transport and synchronization of cilia depend on the distance between the cilia and periodicity. These hydrodynamic properties are investigated below.

Past simulations $[15,42]$ have shown the beat frequency of model cilia to increase in the presence of neighboring cilia. This observation was investigated here by looking at the length of time it takes for the cilia to complete a beat when the inter-ciliary spacing is varied. The cilium was initialized in a vertical position in LR Mode as shown in Fig. 6 and 


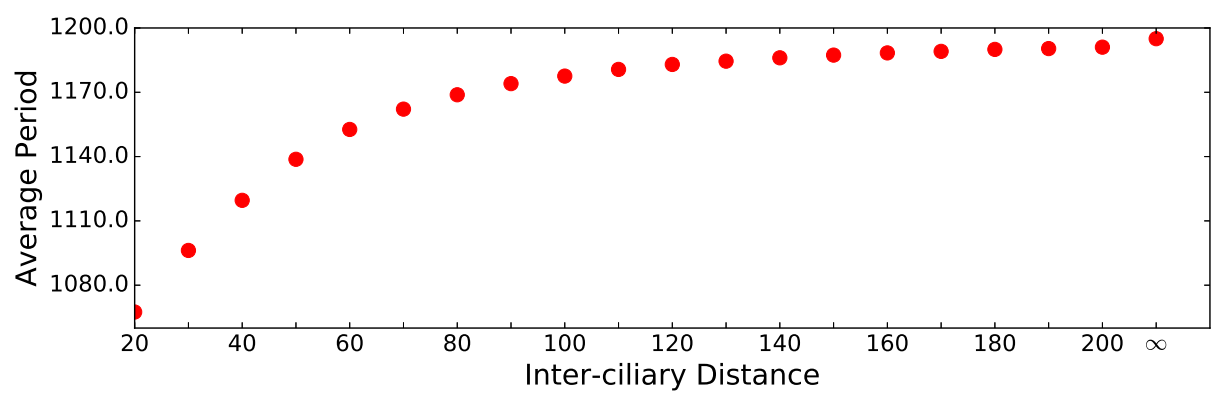

(a) The average period for a single cilium for different inter-ciliary distances, $L$.

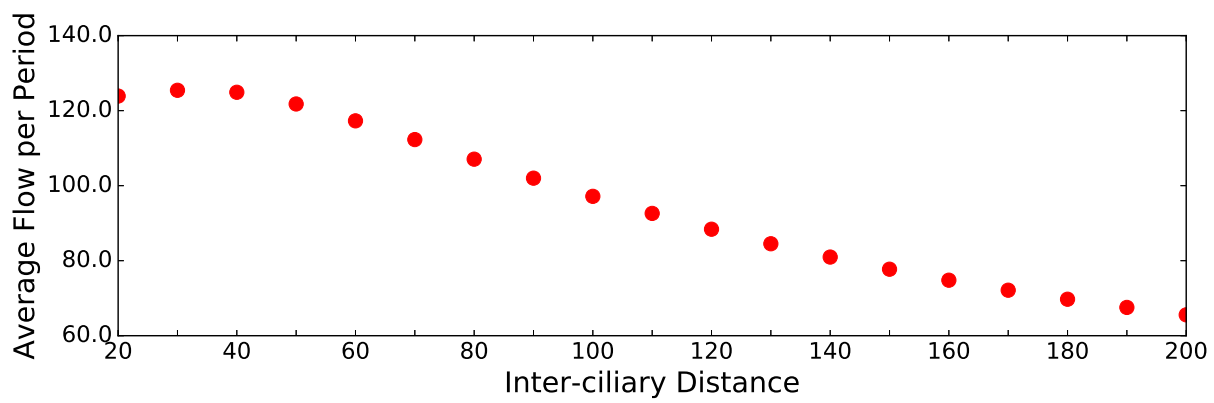

(b) Average net flow per period for periodic cilia with different inter-ciliary distances.

Figure 8: (a) Average period for a single cilium for different inter-ciliary distances, $L$. The last data entry is for the case of an isolated cilium $(L=\infty)$. (b) Average net flow per period for periodic cilia with different inter-ciliary distances. The flow was calculated with numerical integration along a line at the periodic boundary extending from the wall to 4 cilium lengths above the wall. The flow average shown was obtained by averaging over the first 5 complete periods where the cilium was initialized as being completely vertical.

the simulation was run until the end of the 5th power stroke with the period calculated as the average of the final three complete beats. Fig. 8(a) shows a clear trend with the period increasing as the distance between neighboring cilia (the size of the periodic domain) increases. The periods appear to asymptotically approach the period of an isolated cilium, as should be expected.

For the same inter-ciliary distances the net flow was calculated by numerically integrating the flow along the line at the edge of the periodic boundary extending from the wall to a height of 4 cilium lengths above the wall. The flow was integrated in the space dimension with the midpoint rule and a discretization size of 0.25 and integrated across time with a time step of 0.002 . Fig. 8 (b) shows the average net flow per period for different cilia spacing. The fluid displacement decreases as the inter-ciliary distance increases. We conclude from Fig. 8 that cilia packed closer together not only beat faster but they also transport the fluid further each cycle.

Another common interest in cilia is how their beats are coordinated. Much work has shown that hydrodynamic forces are a key component in the synchronization of cilia $[5,29,41,42]$. This is investigated in the present model by looking at two adjacent cilia. 


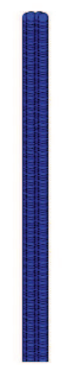

(a)

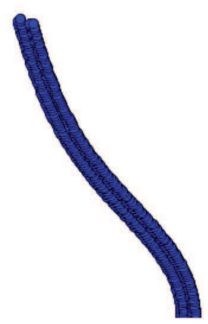

(c)

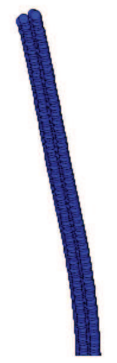

(b)

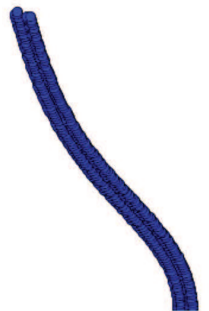

(d)

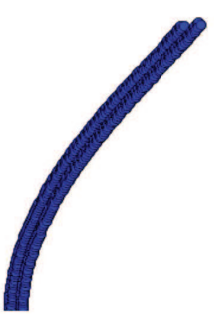

Figure 9: The four initial configurations for two cilia. These configurations can be thought of as starting with a phase lag of $0^{\circ}, 45^{\circ}, 90^{\circ}$ and $180^{\circ}$, respectively. In these cases the length $L$ of the periodic domain equals twice the inter-ciliary distance.

Both two isolated cilia (no periodicity) and a periodic array with two cilia in the periodic box were examined. In these simulations the left and right sliding distances for switching strokes were reduced from 9 and 8 to 5 and 3 respectively. This was helpful in studying synchronicity because it allowed the cilia to be placed closer together without running into each other and because more beats could be completed in a shorter number of time steps, reducing the computational cost. Fig. 7(b) shows this reduced beat.

Synchronicity was studied in the periodic and isolated regimes while varying two parameters: the distance between the two cilia was varied between 10 and 100, and four different initial phase differences were examined that are shown in Fig. 9. The synchronization between the cilia was quantified by computing each cilium's beat period and the lag between the ends of the power strokes of each cilium. The steady state was identified by when these two factors appeared to reach a limit or a steady cycle for the last 25 beats. The beat period and lag were averaged over those final 25 beats. The lag was measured in proportion to the time it took the rightmost cilium to complete one cycle and it is always between $0 \%$ and $50 \%$. A $0 \%$ lag indicates in-phase motion while a $50 \%$ lag indicates anti-phase motion.

Fig. 10 shows snapshots of the velocity field induced by two cilia with an inter-ciliary distance of 15 in the periodic regime with a $180^{\circ}$ initial phase difference. In Fig. 10(a), the cilium on the left and right are both in recovery strokes, and in subsequent snapshots are 


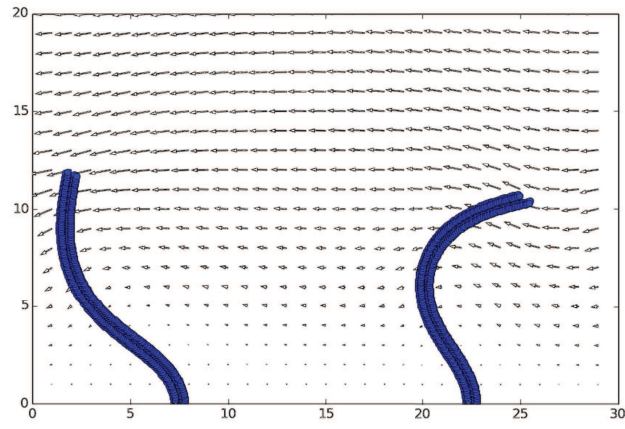

(a)

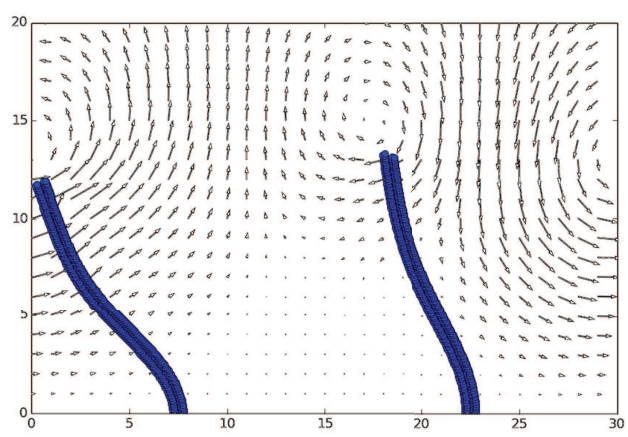

(c)

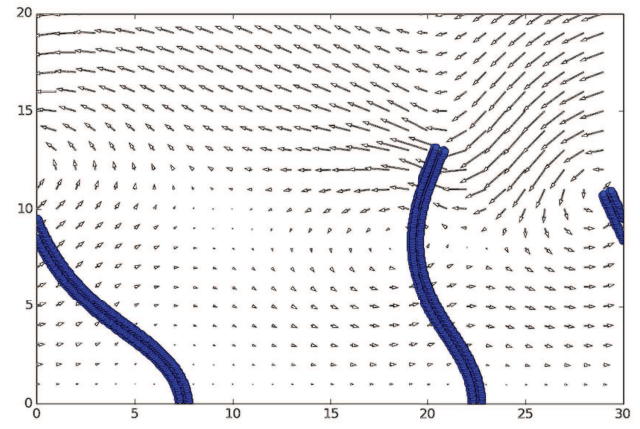

(b)

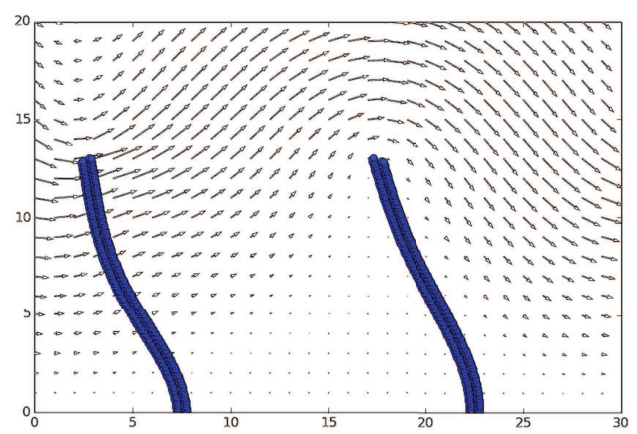

(d)

Figure 10: Four snapshots showing the velocity field induced by two periodic cilia taken 10 non-dimensional time units apart. The cilia were initialized with a $180^{\circ}$ phase difference and an inter-ciliary distance of 15 .

respectively in power and recovery strokes. Note the variety in the velocity fields with mixing clearly exhibited in Figs. 10(b), 10(c), and more monotonic transport in Figs. 10(a), 10(d).

In general two steady states were observed. Either the cilia tended to be nearly in phase, with a lag less than $10 \%$, or nearly out of phase with a lag greater than $40 \%$ (see Fig. 11). In the isolated regime $(L=\infty)$, initial phase differences of $0^{\circ}, 45^{\circ}$ and $90^{\circ}$ all tended toward the in-phase steady state for all inter-ciliary distances; only the initial phase difference of $180^{\circ}$ and inter-ciliary distances larger than 70 , about five cilium lengths, resulted in out-of-phase steady states. The circles in Fig. 11 show the computed lags for the two isolated cilia as a function of inter-ciliary distance for initial phase differences of $45^{\circ}, 90^{\circ}$ and $180^{\circ}$.

On the other hand, the periodic regime exhibited a greater tendency toward the outof-phase steady state. The squares in Fig. 11 show that for the initial phase difference of $45^{\circ}$ (top graph), the in-phase steady state prevailed across all inter-ciliary distances considered. When the initial phase difference was $90^{\circ}$ (middle graph), there was a mix 

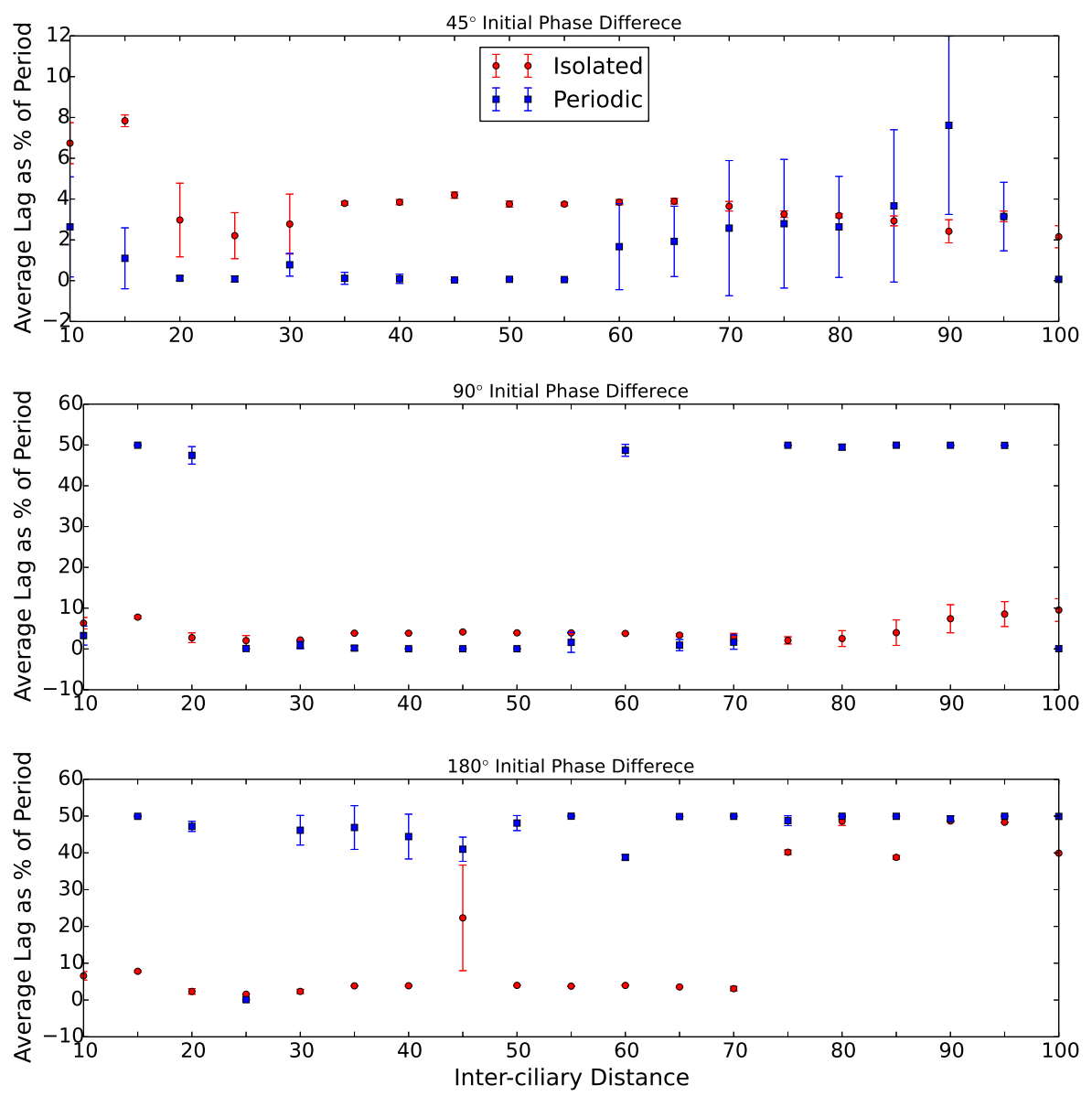

Figure 11: The average lag between two isolated cilia (circles) and two periodic cilia (squares). The top plot corresponds to the cilia initialized with a $45^{\circ}$ phase difference, the middle plot corresponds to the cilia initialized with a $90^{\circ}$ phase difference and the bottom plot corresponds to the cilia initialized with a $180^{\circ}$ phase difference.

of in-phase and out-of-phase steady states. Finally, for the initial phase difference of $180^{\circ}$ (bottom graph), the steady state was out-of-phase with only one exception. The figure also shows that when both the isolated and periodic cases tended to an in-phase steady state, the lags in the periodic case were closer to zero.

Fig. 12 show the average period for the simulations corresponding to Fig. 11. In general, for the periodic regime the figure shows that when the steady state is in-phase, the period is shorter than in the isolated cilia case. Similarly, when the steady state is out-ofphase, the period is longer than in the isolated cilia case. We point out that a cilium may trace a steady state cycle consisting of a shorter beat followed by a longer one. In this case, the two cilia are considered to be in a nearly in-phase steady state as long as the lag between the switching times after the power stroke is within a small percentage of the average period. 

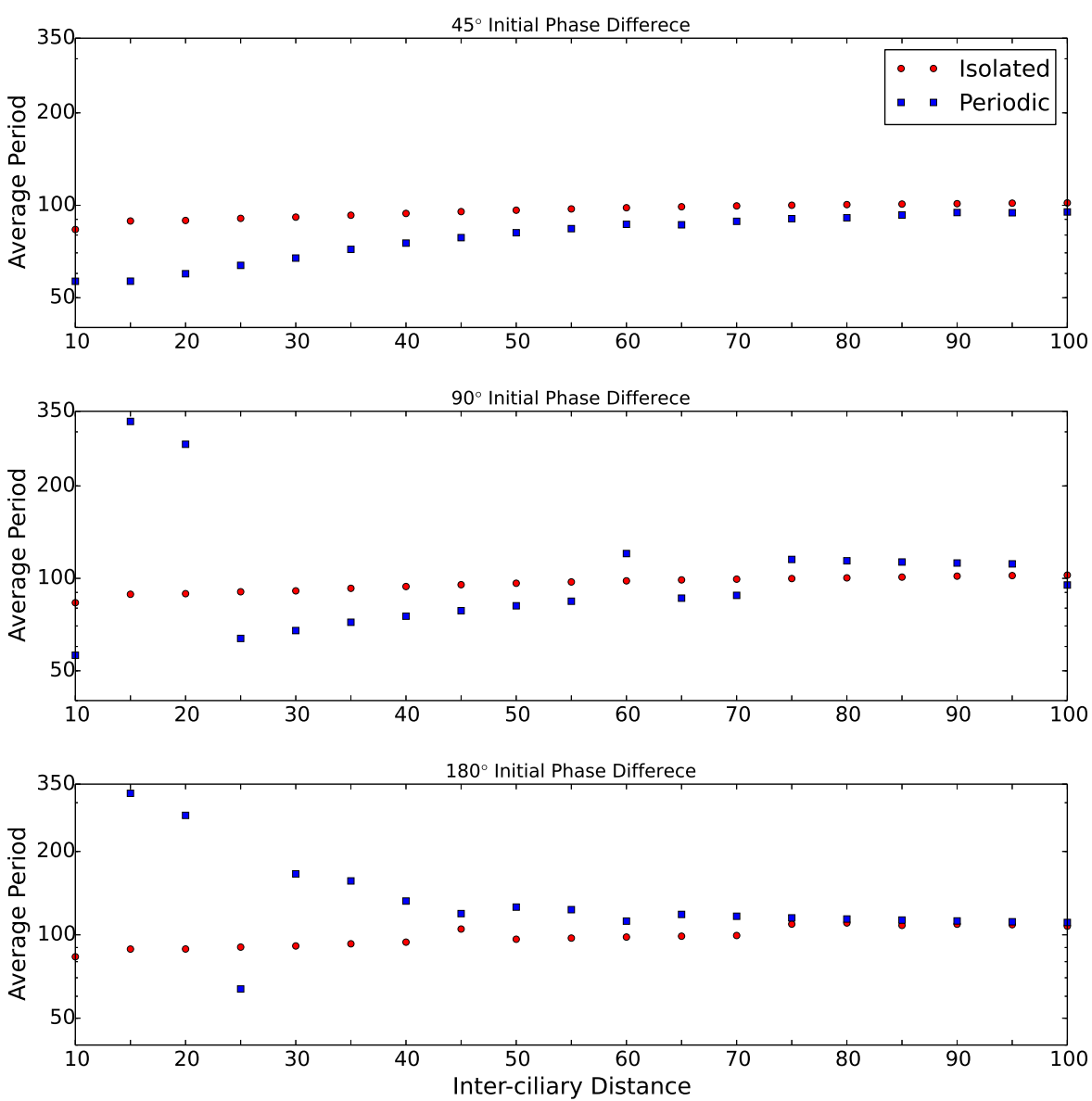

Figure 12: The average period for two isolated cilia (circles) and two periodic cilia (squares). The period shown is the average of the periods of each of the two cilia over the last 25 beats of the simulation. The top plot corresponds to the cilia initialized with a $45^{\circ}$ phase difference, the middle plot corresponds to the cilia initialized with a $90^{\circ}$ phase difference and the bottom plot corresponds to the cilia initialized with a $180^{\circ}$ phase difference.

\section{Conclusions}

We have derived the closed form expression for the fundamental solution of Stokes equations in two space dimensions with periodic boundary conditions in the $x$-direction and a solid plane wall at $y=0$. To accommodate the no-slip condition on the wall, the system of images was used. The formulation has been derived in the context of regularized Stokeslets; however, the expression for the traditional (singular) Stokeslet is easily recovered by taking the limit as the regularization parameter approaches zero. Applying this formulation to compute the swimming speed of an infinite waving sheet and comparing the result with Taylor's analytic expression for small amplitude waves provided a validating example of our method, which is not restricted to small amplitude waves. A second example of a cylinder moving parallel and perpendicular to the wall provided 
further validation of the method and showed that the net force for the parallel motion is smaller for smaller periodic domains. The net force for the perpendicular motion was insensitive to the periodic domain size.

The usefulness of the result was further demonstrated by applying it to compute the flow generated by cilia. The cilium model was adapted from [13] in which dyneins from one microtubule attach and detach to different sites in the other microtubule. This mechanism applied asymmetrically generates a cilium beat that is not prescribed a priori and produces particle transport. Several properties of cilia such as synchronization and effective fluid transport were investigated in terms of their dependence on the spacing between individual cilia in both periodic and isolated regimes.

The computational method developed for singly-periodic flows in two dimensions bounded by a plane is based on the explicit Green's function. The closed-form formula for the velocity makes the method easy to implement as an $N$-body problem where each force contributes to the velocity of all points of the cilia. A limitation of our fluid model is that while the formulation is possible in two dimensions, it does not extend to three dimensions in a straightforward way, where no closed-form expression for the doublyperiodic Stokeslet is available and the models have to resort to fast summation techniques to evaluate series solutions $[9,18,24,25,31,40]$. The cilium model in two dimensions is limited to two microtubule doublets and can be thought of as representing the motion of "cilium sheets" extending infinitely into the paper. For this reason, the fluid velocities in between two cilia tend to have small magnitudes (see e.g. Fig. 10(c)-(d)) compared to three dimensions where the flow can go around the cilia and develop more substantive velocities between cilia. In spite of the limitations, the two-dimensional model displays a rich set of relevant behaviors and provides insights transferrable to three dimensions related to cilia beat synchronization and the emergent dynamics based on the switching mechanism between power and recovery strokes.

\section{Acknowledgments}

The work of the authors was supported in part by the National Science Foundation (NSF) Grant No. DMS-1043626.

\section{References}

[1] J. Ainley, S. Durkin, R. Embid, P. Boindala, and R. Cortez. The method of images for regularized Stokeslets. Journal of Computational Physics, 227:4600-4616, 2008.

[2] Gregory R. Baker and Lan D. Pham. A comparison of blob methods for vortex sheet roll-up. J. Fluid Mech., 547:297-316, 2006.

[3] J. R. Blake. A note on the image system for a Stokeslet in a no-slip boundary. Proceedings of the Cambridge Philosophical Society. Mathematical and Physical Sciences, 70:303-310, 1971.

[4] J Bleibel. Ewald sum for hydrodynamic interactions with periodicity in two dimensions. Journal of Physics A: Mathematical and Theoretical, 45(22):225002, 2012. 
[5] Douglas R Brumley, Marco Polin, Timothy J Pedley, and Raymond E Goldstein. Metachronal waves in the flagellar beating of volvox and their hydrodynamic origin. Journal of The Royal Society Interface, 12(108):20141358, 2015.

[6] Spencer H Bryngelson and Jonathan B Freund. Buckling and its effect on the confined flow of a model capsule suspension. Rheologica Acta, pages 1-14, 2016.

[7] Duanduan Chen, Jun Ren, Yuqian Mei, and Yuanqing Xu. The respiratory ciliary motion produced by dynein activity alone: A computational model of ciliary ultrastructure. Technology and Health Care, 23(s2):S577-S586, 2015.

[8] R. Cortez. The method of regularized stokeslets. SIAM Journal on Scientific Computing, 23((4)):1203-1225, 2001.

[9] Ricardo Cortez and Franz Hoffmann. A fast numerical method for computing doublyperiodic regularized stokes flow in 3d. Journal of Computational Physics, 258:1 - 14, 2014.

[10] Ricardo Cortez and Douglas Varela. A general system of images for regularized stokeslets and other elements near a plane wall. Journal of Computational Physics, 285:41-54, March 2015.

[11] Darren G. Crowdy and Yizhar Or. Two-dimensional point singularity model of a lowreynolds-number swimmer near a wall. Phys. Rev. E, 81:036313, Mar 2010.

[12] Joseph M. Crowley. Viscosity-induced instability of a one-dimensional lattice of falling spheres. Journal of Fluid Mechanics, 45(1):151159, Jan 1971.

[13] Robert H. Dillon and Lisa Fauci. An integrative model of internal axoneme mechanics and external fluid dynamics in ciliary beating. Journal of Theoretical Biology, 207:175-191, 2000.

[14] ML Ekiel-Jeżewska and BU Felderhof. Periodic sedimentation of three particles in periodic boundary conditions. Physics of Fluids, 17(9):093102, 2005.

[15] S. Gueron, K. Levit-Gurevich, N. Liron, and J. Blum. Cilia internal mechanism and metachronal coordination as the result of hydrodynamical coupling. Proc. Natl. Acad. Sci., 94(12):6001-6006, 1997.

[16] Shay Gueron and Konstantin Levit-Gurevich. A three-dimensional model for ciliary motion based on the internal 9+ 2 structure. Proceedings of the Royal Society of London B: Biological Sciences, 268(1467):599-607, 2001.

[17] Brian M Haines, Igor S Aranson, Leonid Berlyand, and Dmitry A Karpeev. Effective viscosity of dilute bacterial suspensions: a two-dimensional model. Physical biology, 5(4):046003, 2008.

[18] H. Hasimoto. On the periodic fundamental solutions of the stokes equations and their application to viscous flow past a cubic array of spheres. Journal of Fluid Mechanics, 5(2):317328, Feb 1959.

[19] David A. Hoey, Shane Tormey, Stacy Ramcharan, Fergal J. O'Brien, and Christopher R. Jacobs. Primary cilia-mediated mechanotransduction in human mesenchymal stem cells. STEM CELLS, 30(11):2561-2570, 2012.

[20] PG Jayathilake, Zhijun Tan, DV Le, HP Lee, and BC Khoo. Three-dimensional numerical simulations of human pulmonary cilia in the periciliary liquid layer by the immersed boundary method. Computers \& Fluids, 67:130-137, 2012.

[21] D. J. JEFFREY and Y. ONISHI. The slow motion of a cylinder next to a plane wall. The Quarterly Journal of Mechanics and Applied Mathematics, 34(2):129-137, 1981.

[22] Dae Kyung Kim, Ji Yeon Hyun, Sung Chul Kim, Han Sung Kim, and Sei Young Lee. Inertial effects on cylindrical particle migration in linear shear flow near a wall. Microfluidics and Nanofluidics, 20(5):1-10, 2016.

[23] WL Lee, PG Jayathilake, Zhijun Tan, DV Le, HP Lee, and BC Khoo. Muco-ciliary transport: 
effect of mucus viscosity, cilia beat frequency and cilia density. Computers \& Fluids, 49(1):214221, 2011.

[24] Dag Lindbo and Anna-Karin Tornberg. Spectrally accurate fast summation for periodic stokes potentials. J. Comput. Physics, 229(23):8994-9010, 2010.

[25] Dag Lindbo and Anna-Karin Tornberg. Spectral accuracy in fast ewald-based methods for particle simulations. J. Comput. Physics, 230(24):8744-8761, 2011.

[26] Charles B. Lindemann and Kathleen A. Lesich. Flagellar and ciliary beating: the proven and the possible. Journal of Cell Science, 123:519-528, 2010.

[27] Gary R. Marple, Alex Barnett, Adrianna Gillman, and Shravan Veerapaneni. A fast algorithm for simulating multiphase flows through periodic geometries of arbitrary shape. SIAM Journal on Scientific Computing, 38(5):B740-B772, 2016.

[28] Sorin M Mitran. Metachronal wave formation in a model of pulmonary cilia. Computers $\mathcal{E}$ structures, 85(11):763-774, 2007.

[29] Babak Nasouri and Gwynn J Elfring. Hydrodynamic interactions of cilia on a spherical body. Physical Review E, 93(3):033111, 2016.

[30] C. Pozrikidis. Boundary integral and singularity methods for linearized viscous flow. Cambridge University Press, 1992.

[31] Constantine Pozrikidis. Computation of periodic green's functions of stokes flow. J. Eng. Math., 30:79-96, 1996.

[32] P.G. Saffman. Vortex Dynamics. Cambridge University Press, 1992.

[33] David Saintillan, Eric Darve, and Eric SG Shaqfeh. A smooth particle-mesh ewald algorithm for stokes suspension simulations: The sedimentation of fibers. Physics of Fluids (1994present), 17(3):033301, 2005.

[34] David Saintillan and Michael J Shelley. Instabilities, pattern formation, and mixing in active suspensions. Physics of Fluids (1994-present), 20(12):123304, 2008.

[35] Julie Simons, Sarah Olson, Ricardo Cortez, and Lisa Fauci. The dynamics of sperm detachment from epithelium in a coupled fluid-biochemical model of hyperactivated motility. Journal of theoretical biology, 354:81-94, 2014.

[36] James W. Swan and Gang Wang. Rapid calculation of hydrodynamic and transport properties in concentrated solutions of colloidal particles and macromolecules. Physics of Fluids, 28(1), 2016.

[37] Prue Talbot, Christine Geiske, and Michael Knoll. Oocyte pickup by the mammalian oviduct. Molecular biology of the cell, 10(1):5-8, 1999.

[38] Geoffrey Taylor. Analysis of the swimming of microscopic organisms. Proceedings of the Royal Society of London A: Mathematical, Physical and Engineering Sciences, 209(1099):447-461, 1951.

[39] Ann E Tilley, Matthew S Walters, Renat Shaykhiev, and Ronald G Crystal. Cilia dysfunction in lung disease. Annual review of physiology, 77:379-406, 2014.

[40] Anna-Karin Tornberg. The ewald sums for singly, doubly and triply periodic electrostatic systems. Adv. Comput. Math., 42(1):227-248, 2016.

[41] Andrej Vilfan and Frank Jülicher. Hydrodynamic flow patterns and synchronization of beating cilia. Physical review letters, 96(5):058102, 2006.

[42] Xingzhou Yang, Robert H. Dillon, and Lisa J. Fauci. An integrative computational model of multiciliary beating. Bull Math Biol, 70(4):1192-215, 2008.

[43] AA Zick and GM Homsy. Stokes flow through periodic arrays of spheres. Journal of fluid mechanics, 115:13-26, 1982. 